\title{
Thermal conductivity of amorphous carbon thin films
}

\author{
Andrew J. Bullen, Keith E. O'Hara, and David G. Cahill ${ }^{\text {a) }}$ \\ Department of Materials Science and Engineering, Coordinated Science Laboratory, \\ and Materials Research Laboratory, University of Illinois, Urbana, Illinois 61801 \\ Othon Monteiro \\ Lawrence Berkeley Laboratory, University of California, Berkeley, California 94720
}

Achim von Keudell

Max-Planck-Intitut für Plasmaphysik, D-85740 Garching bei München, Germany

(Received 30 May 2000; accepted for publication 5 August 2000)

\begin{abstract}
Thermal conductivities $\Lambda$ of amorphous carbon thin films are measured in the temperatures range $80-400 \mathrm{~K}$ using the $3 \omega$ method. Sample films range from soft $a-\mathrm{C}: \mathrm{H}$ prepared by remote-plasma deposition $\left(\Lambda=0.20 \mathrm{~W} \mathrm{~m}^{-1} \mathrm{~K}^{-1}\right.$ at room temperature) to amorphous diamond with a large fraction of $s p^{3}$ bonded carbon deposited from a filtered-arc source $\left(\Lambda=2.2 \mathrm{~W} \mathrm{~m}^{-1} \mathrm{~K}^{-1}\right)$. Effective-medium theory provides a phenomenological description of the variation of conductivity with mass density. The thermal conductivities are in good agreement with the minimum thermal conductivity calculated from the measured atomic density and longitudinal speed of sound. (C) 2000 American Institute of Physics. [S0021-8979(00)07221-2]
\end{abstract}

\section{INTRODUCTION}

Amorphous carbon $(a-C)$ exists in an amazing variety of forms with microstructures and physical properties that depend sensitively on preparation method. ${ }^{1}$ Because $a$-C thin films are often used as protective coatings, the most thoroughly studied of these structure-property relationships are the dependence of the mechanical properties, e.g., elastic constants and hardness, on deposition conditions, atomic density, and hydrogen content. The focus of our experimental study, thermal conductivity, like the mechanical properties, derives from the bonding and geometry of the atomic lattice. The large variability of microstructures within this single class of materials provides a unique opportunity for exploring heat transport in disordered solids $\mathrm{s}^{2,3}$ and the applicability of the minimum thermal conductivity ${ }^{4,5}$ to materials with heterogeneous microstructures that are common in thin films. ${ }^{6,7}$ But we also anticipate that these new data will provide valuable insights on the high and low conductivities that can be produced in thin film $a$-C for applications in the thermal engineering of microdevices. ${ }^{8,9}$

The concept of a "minimum thermal conductivity" $\Lambda_{\min }$ is based on a theory of heat transport originally proposed by Einstein: ${ }^{10}$ the atomic vibrations are assumed to be incoherent and therefore heat diffuses between the Einstein oscillators on a time scale of $1 / 2$ the period of vibration. Einstein's theory could not explain the large thermal conductivities of most crystalline dielectrics but his and related models $s^{4,5,11}$ are useful for understanding the thermal conductivity of amorphous materials and crystals with certain types of strong disorder.

We include larger oscillating entities than the single atoms considered by Einstein by borrowing from the Debye model of lattice vibrations and dividing the sample into re-

\footnotetext{
${ }^{a)}$ Electronic mail: d-cahill@uiuc.edu
}

gions of size $\lambda / 2$, where $\lambda$ is an acoustic wavelength, and whose frequencies of oscillation are given by the low frequency speed of sound $\omega=2 \pi v / \lambda .^{5,7}$

$$
\Lambda_{\min }=\left(\frac{\pi}{6}\right)^{1 / 3} k_{B} n^{2 / 3} \sum_{i=1}^{3} v_{i}\left(\frac{T}{\Theta_{i}}\right)^{2} \int_{0}^{\Theta_{i} / T} \frac{x^{3} e^{x}}{\left(e^{x}-1\right)^{2}} d x .
$$

The index $i$ labels the three sound modes (two transverse and one longitudinal) with speeds of sound $v_{i} ; \Theta_{i}$ is the cutoff frequency for each polarization expressed in Kelvin, $\Theta_{i}$ $=v_{i}\left(\hbar / k_{B}\right)\left(6 \pi^{2} n\right)^{1 / 3}$, and $n$ is the number density of atoms. This model has no free parameters and is in good agreement with data for a wide variety of bulk disordered materials near room temperature. ${ }^{5}$

Since diamond has the largest values of $n$ and $v_{i}{ }^{12}$ of any material, the high temperature limit of $\Lambda_{\text {min }}$ also has the largest possible value. Figure 1 shows the calculated $\Lambda_{\text {min }}$ for diamond with comparisons to previously published data for amorphous carbon. ${ }^{713-15}$ Data for bulk samples of high-dose neutron-irradiated diamond ${ }^{13}$ and disordered carbon produced by high-pressure conversion of $\mathrm{C}_{60}{ }^{15}$ were measured by traditional steady-state methods; the conductivities of thin film samples were measured using the mirage effect ${ }^{14}$ and picosecond thermoreflectance. ${ }^{7}$ The thin film data were measured only for room temperature, and therefore the unusual temperature of the two bulk samples cannot be confirmed in the thin film samples. Furthermore, while picosecond reflectance is a powerful probe of elastic properties and interfacial transport of acoustic and thermal energy, measurements of thermal conductivity using this method are relatively indirect and require assumptions about the heat capacity of the films. ${ }^{7}$

\section{EXPERIMENTAL DETAILS}

Thin film samples of $a$-C:H were prepared at the MaxPlanck-Institut für Plasmaphysik by remote-plasma chemical vapor deposition (RPCVD)—chosen to produce a soft, low- 


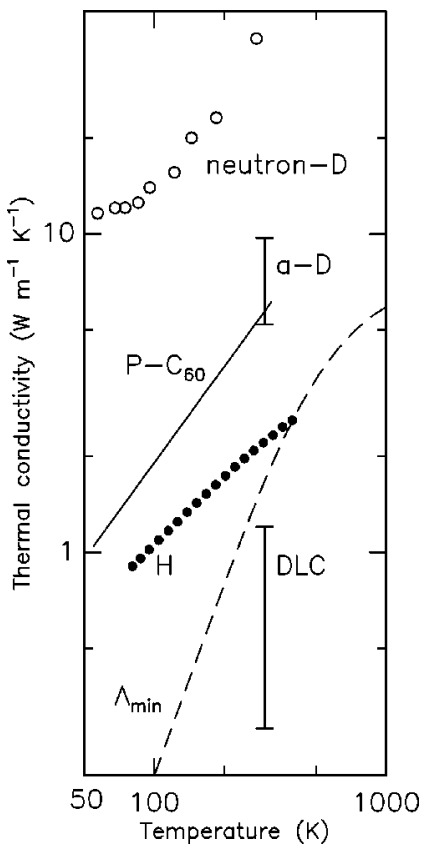

FIG. 1. Summary of published data for amorphous carbon with comparison to the minimum thermal conductivity $\Lambda_{\min }$ calculated for diamond (dashed line) and the highest thermal conductivity measured in this work (sample $\mathrm{H}$, filled circles, see description in Table I); (i) neutron-D, diamond irradiated by neutrons at $2 \times 10^{22} \mathrm{~cm}^{-2}$ Ref. 13; (ii) $\mathrm{P}-\mathrm{C}_{60}$, high-pressure conversion of $\mathrm{C}_{60}$, Ref. 15; (iii) DLC, range of conductivities measured in diamondlike carbon thin films at room temperature, Refs. 7 and 14; (iv) $a$-D, range of conductivities measured in amorphous diamond films at room temperature, Ref. 7.

density form of $a-\mathrm{C}: \mathrm{H}$ - and by plasma-assisted CVD (PACVD); the PACVD samples have mechanical properties that are typical for protective coatings of "diamond-likecarbon", (DLC). ${ }^{16}$ Carbon-to-hydrogen ratios measured on similar samples are 1:1 for RPCVD and 2:1 for PACVD. Additional samples of DLC films were obtained from Delphi Automotive Systems and Surmet Corporation. At Lawrence Berkeley Laboratory, $a$-C films were deposited by filteredarc deposition (FAD) ${ }^{17-19}$ using two acceleration voltages: 100 and $2000 \mathrm{~V}$. The fractions of $s p^{3}$ bonded carbon measured by $\mathrm{EELS}^{18}$ on similar samples are $80 \%$ at $100 \mathrm{~V}$ bias and $30 \%$ at $2000 \mathrm{~V}$ bias; $a$-C films with low concentrations of hydrogen and carbon bonding dominated by $s p^{3}$ hybridization are often referred to as "amorphous diamond" ( $a$-D) or "tetrahedrally-bonded" amorphous carbon (ta-C).

We use the $3 \omega$ method $^{20,21}$ to measure the thermal conductivity of $a$-C films in the temperature range $80<T<400$ K. A $10 \mu \mathrm{m}$ wide Al line-sputter deposited on the surface of the sample and patterned by photolithography—serves as both the heater and the thermometer in the measurement. If the film thickness $h$ is small compared to the width of the metal line, heat flow is one dimensional in the thin film and two dimensional (radial) in the substrate. ${ }^{21}$ Also, as long as $h$ is small compared to the penetration depth of the thermal waves, the thin film simply adds a frequency-independent temperature oscillation to the known thermal response of the substrate. Most of our $\mathrm{a}$-C samples were deposited on $\mathrm{Si}$ substrates with a $100 \mathrm{~nm}$ thick layer of thermally grown $\mathrm{SiO}_{2}$, which is needed to improve the electrical isolation
TABLE I. Deposition parameters and physical properties of $a$-C films. Films are deposited by plasma-assisted CVD, remote-plasma CVD, and filtered-arc deposition. Films were deposited at the Max-Planck-Institut für Plasmaphysik (A,B,C,D), and Lawrence Berkeley Laboratory (H,I,L,M). Additional samples were obtained from the Surmet Corporation $(F, G)$ and Delphi Automotive Systems (E).

\begin{tabular}{lccccr}
\hline \hline Sample & $\begin{array}{c}\text { Film thickness } \\
(\mathrm{nm})\end{array}$ & $\begin{array}{c}\text { Density } \\
\left(\mathrm{g} \mathrm{cm}^{-3}\right)\end{array}$ & $\begin{array}{c}v_{l} \\
\mathrm{~km} \mathrm{~s}^{-1}\end{array}$ & Method & $\begin{array}{c}\text { Bias } \\
(\mathrm{V})\end{array}$ \\
\hline A,B & 94,313 & 1.8 & 8.7 & PACVD & 200 \\
C,D & 108,325 & 0.9 & 3.4 & RPCVD & 15 \\
E & 3800 & 2.1 & - & PACVD & 450 \\
F & 120 & 1.2 & - & PACVD & 0 \\
G & 280 & 1.7 & - & PACVD & 0 \\
H,I & 47,592 & 2.8 & 14.0 & FAD & 100 \\
L,M & 19,565 & 2.3 & 12.7 & FAD & 2000 \\
\hline \hline
\end{tabular}

between the $\mathrm{Si}$ substrates and the $\mathrm{Al}$ metallization. The added thermal resistance of the $\mathrm{SiO}_{2}$ layer is measured separately and subtracted from the raw data. ${ }^{21}$

Conversion of the measured thermal resistance to thermal conductivity requires accurate measurements of film thickness $h$. We measure $h$ using spectroscopic variableangle ellipsometry; the optical properties of the $a-\mathrm{C}$ films are modeled using a fit to the resonant frequency, oscillator strength, and damping of two Lorentz-oscillators. Alternatively, e.g., if the optical modeling produced a poor fit to the ellipsometry data, we use scanning electron microscopy of a fracture cross section to measure $h$. Areal densities of carbon are measured using Rutherford backscattering spectrometry of the stopping power of the $a-\mathrm{C}$ film. The combination of areal density and $h$ gives the film density, see (Table I). We measure longitudinal speeds of sound $v_{l}$ by "picosecond ultrasonics:"7 an Al thin-film transducer produces and detects acoustic waves generated by a mode-locked Ti:sapphire laser operating at $780 \mathrm{~nm}$; values for $v_{l}$ are listed in Table I.

\section{RESULTS AND DISCUSSION}

Figure 2 shows the results of our thermal conductivity measurements. In all cases, the thermal conductivity has the temperature dependence expected for an amorphous solid in this temperature range. ${ }^{5}$ In four cases, we measured the same type of film for two values of the thickness $h$ to determine the effects of the finite thermal conductance of interfaces on our measurements. ${ }^{21}$ For the relatively low conductivities of the RPCVD and PACVD films [see Fig. 2(a)], the interface effects have little effect on the measured conductivity of films with $h \sim 100 \mathrm{~nm}$. Interface effects are more pronounced in the FAD films [see Fig. 2(b)]. Using the assumption that the true conductivity of the film is independent of film thickness, we can separate the true conductivity of the film from the interface thermal conductance; ${ }^{21}$ for both sets of FAD films shown in Fig. 2(b), the true conductivity is $\approx 15 \%$ larger than the measured conductivity of the thicker film.

We have discovered that effective medium theory ${ }^{22}$ provides a surprisingly good description of the variation of conductivity with mass density. ${ }^{23}$ The conductivity of a composite structure made of a matrix material and spherical inclusions of a second phase is given $b^{22}$ 

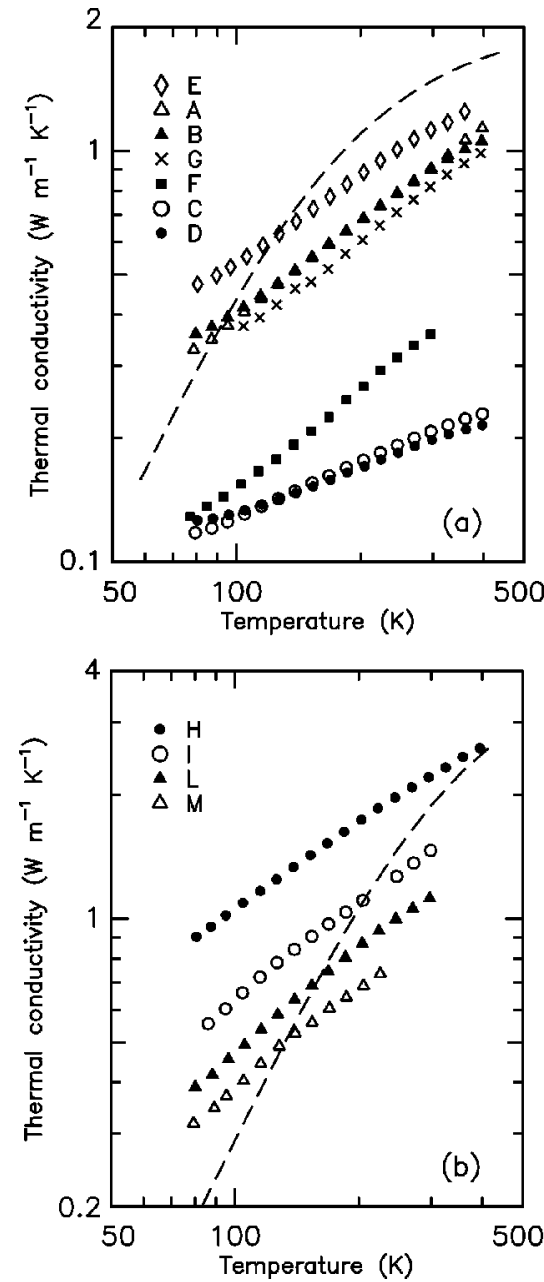

FIG. 2. Thermal conductivity of $a$-C films deposited by: (a) plasma-assisted CVD and (b) filtered-arc deposition. Data symbols are labeled by a letter that identifies each sample; see Table I for sample descriptions; $\Lambda_{\text {min }}$ for samples $\mathrm{A}$ and $\mathrm{H}$ are shown as dashed lines in (a) and (b), respectively.

$$
\begin{aligned}
4 \Lambda= & \left(3 f_{2}-1\right) \Lambda_{2}+\left(3 f_{1}-1\right) \Lambda_{1} \\
& +\left[\left(\left(3 f_{2}-1\right) \Lambda_{2}+\left(3 f_{1}-1\right) \Lambda_{1}\right)^{2}+8 \Lambda_{1} \Lambda_{2}\right]^{1 / 2},
\end{aligned}
$$

where $\Lambda_{1}$ is the conductivity of the matrix, $\Lambda_{2}$ is the conductivity of the second phase, and $f_{1}, f_{2}$ are the volume fractions of the matrix and second phase, respectively. Figure 3 compares the predictions of this theory to the room temperature conductivity of $a$-C films. The theory fits the data reasonably well and enables us to extrapolate the conductivity to the full density of diamond, $\Lambda=4.0 \mathrm{~W} \mathrm{~m}^{-1} \mathrm{~K}^{-1}$.

Experiments on $a$-C have often been interpreted in terms of heterogeneous microstructures, ${ }^{24,25}$ but the accuracy and generality of these various microstructural models remains controversial. Our two-component model, see Eq. (2) and Fig. 3, for the thermal conductivity is probably an oversimplification of the true complexity of $a$-C microstructures. Nevertheless, we believe this phenomenological model will be a useful engineering guide for predicting the conductivity of $a$-C films when only the density is known.

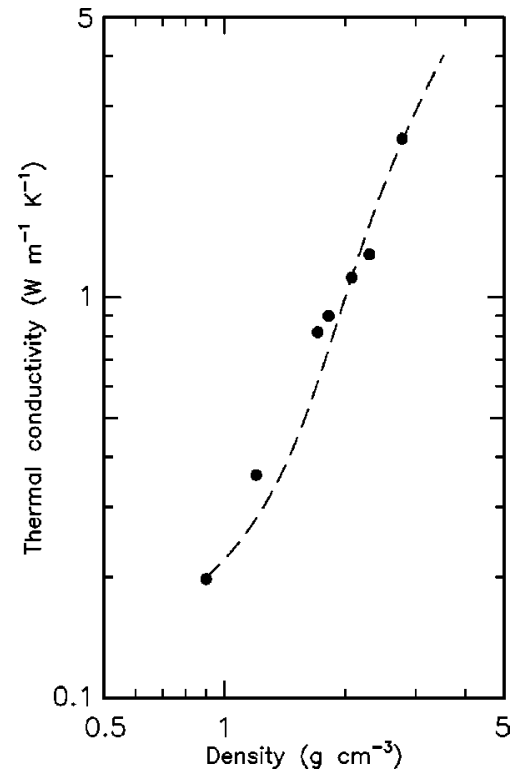

FIG. 3. Room temperature thermal conductivity of $a$-C thin films as a function of mass density. The thermal conductivity of the filtered-arc deposited $a$-C has been adjusted by a small factor $(\approx 15 \%)$ to correct for the finite interface conductance. The dashed line is an effective medium calculation [Eq. (2)] using two components; component 1 has the conductivity and density of our lowest conductivity film and component 2 has the density of diamond $\rho=3.51 \mathrm{~g} \mathrm{~cm}^{-3}$. The conductivity of component 2 is adjusted to fit the data: $\Lambda_{2}=4.0 \mathrm{~W} \mathrm{~m}^{-1} \mathrm{~K}^{-1}$.

Figure 2 also includes comparisons of the data to the calculated $\Lambda_{\min }$ for samples A (DLC prepared by PACVD) and $\mathrm{H}$ ("amorphous diamond" prepared by FAD). The temperature dependence of $\Lambda_{\text {min }}$ is steeper than the data; this result is generally observed for both bulk ${ }^{5}$ and thin film ${ }^{6}$ amorphous materials since Eq. (1) does not include contributions to the heat transport by low frequency phonons with long mean-free-paths. ${ }^{3}$ But in the high temperature limit, the agreement is good, particularly for sample H [see Fig. 2(b)].

The agreement between the measured and calculated conductivities is made more explicit in Fig. 4 where we com-

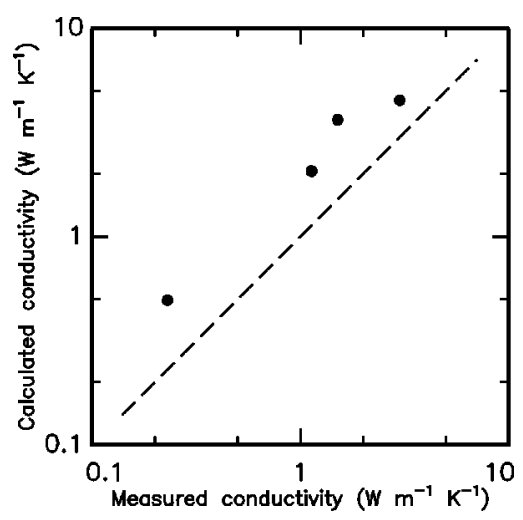

FIG. 4. Comparison of the thermal conductivity at $400 \mathrm{~K}$ for samples $\mathrm{C}, \mathrm{A}$, $\mathrm{L}$, and $\mathrm{H}$ and the high temperature limit of Eq. (1); $\Lambda_{\min }=0.40 k_{B} n^{2 / 3}\left(v_{l}\right.$ $+2 v_{t}$ ). Longitudinal speeds of sounds $v_{l}$ are measured by picosecond ultrasonics (see Table I); we assume $v_{t} \approx 0.60 v_{l}$ (corresponding to a Poisson's ratio of 0.22 ) and ignore the contribution of hydrogen to the atomic densities $n$. The dashed line indicates perfect agreement between measured and calculated values. 
pare the high temperature limit of $\Lambda_{\min }$ to the data at $400 \mathrm{~K}$, the highest temperature of our measurements. (Data for sample $\mathrm{L}$ are restricted to $T<300 \mathrm{~K}$ because of stray electrical conductance at higher temperatures. In this case, we have extrapolated the data to $400 \mathrm{~K}$ using the temperature dependence of sample $\mathrm{H}$.) The calculations reproduce the trend in the data well; we note, however, that the calculated conductivities are consistently greater than the measured values. The fact that the thermal conductivities are increasing with temperature contributes to this discrepancy; measurements at higher temperatures would show better agreement with the model. For the lowest conductivity films, however, the temperature dependence of the data is relatively weak and the calculated conductivity exceeds the measured value at $400 \mathrm{~K}$ by a factor of $\approx 2$. This relatively large difference between measured and calculated conductivity is also observed in amorphous Se. ${ }^{5}$ But given the simplifying assumptions of the model, ${ }^{5}$ disagreements of this magnitude are expected and we conclude that the minimum thermal conductivity calculated from the mean atomic densities and speeds of sound provides an adequate description of heat transport in a wide variety of $a-\mathrm{C}$ thin film materials.

\section{ACKNOWLEDGMENTS}

The authors thank Don Morelli and the Surmet Corp. for providing additional samples of DLC. This work was supported by NSF Grant No. CTS 99-78822. Sample characterization by RBS, SEM, ellipsometry, and picosecond ultrasonics used the facilities of the Center for Microanalysis of Materials, supported by U.S. DOE Grant No. DEFG02-96ER45439.
${ }^{1}$ J. Robertson, Adv. Phys. 35, 317 (1986); Prog. Solid State Chem. 21, 199 (1991).

${ }^{2}$ R. Orbach, Philos. Mag. B 65, 289 (1992).

${ }^{3}$ J. L. Feldman, P. B. Allen, and S. R. Bickham, Phys. Rev. B 59, 3551 (1999).

${ }^{4}$ G. A. Slack, in Solid State Physics: Advances in Research and Applications, edited by F. Seitz and A. G. Turnball (Academic, New York, 1979), Vol. 34, p. 1.

${ }^{5}$ D. G. Cahill, S. K. Watson, and R. O. Pohl, Phys. Rev. B 46, 6131 (1992).

${ }^{6}$ S.-M. Lee, D. G. Cahill, and T. H. Allen, Phys. Rev. B 52, 253 (1995).

${ }^{7}$ C. J. Morath, H. J. Maris, J. J. Cuomo, D. L. Pappas, A. Grill, V. V. Patel, J. P. Doyle, and K. L. Saenger, J. Appl. Phys. 76, 2636 (1994).

${ }^{8}$ K. E. Goodson, Y.-S. Ju, and M. Asheghi, in Microscale Energy Transport, edited by C. L. Tien, A. Majumdar, and F. M. Gerner (Taylor \& Francis, New York, 1998), pp. 229-293.

${ }^{9}$ K. E. Goodson and Y. Sugtaek Ju, Annu. Rev. Mater. Sci. 29, 261 (1999).

${ }^{10}$ A. Einstein, Ann. Phys. (Leipzig) 35, 679 (1911).

${ }^{11}$ C. Kittel, Phys. Rev. 75, 972 (1948).

${ }^{12}$ H. J. McSkimin and W. L. Bond, Phys. Rev. 105, 116 (1957).

${ }^{13}$ D. T. Morelli, T. A. Perry, J. W. Vandersande, and C. Uher, Phys. Rev. B 48, 3037 (1993)

${ }^{14}$ W. Hurler, M. Pietralla, and A. Hammerschmidt, Diamond Relat. Mater. 4, 954 (1995).

${ }^{15}$ A. Smontara, K. Biljakovic, D. Staresinic, D. Pajic, M. E. Kozlov, M. Hirabayashi, M. Tokumoto, and H. Ihara, Physica B 219\&220, 160 (1996).

${ }^{16}$ J. W. Zou, K. Reichelt, K. Schmidt, and B. Dischler, J. Appl. Phys. 65, 3914 (1989).

${ }^{17}$ S. Aisenberg and R. Chabot, J. Appl. Phys. 42, 2953 (1971).

${ }^{18}$ G. M. Pharr et al., Appl. Phys. Lett. 68, 779 (1996).

${ }^{19}$ P. J. Fallon, V. S. Veerasamy, C. A. Davis, J. Robertson, G. A. J. Amaratunga, W. I. Milne, and J. Koskinen, Phys. Rev. B 48, 4777 (1993).

${ }^{20}$ D. G. Cahill, Rev. Sci. Instrum. 61, 802 (1990).

${ }^{21}$ S.-M. Lee and D. G. Cahill, J. Appl. Phys. 81, 2590 (1997).

${ }^{22}$ R. Landauer, J. Appl. Phys. 23, 779 (1952).

${ }^{23}$ Effective medium theories have often been used for modeling the optical properties of $a$-C; see, for an early example, F. W. Smith, J. Appl. Phys. 55, 764 (1984).

${ }^{24}$ J. Robertson and E. P. O’Reilly, Phys. Rev. B 35, 2946 (1987).

${ }^{25}$ C. Jäger, J. Gottwald, H. W. Spiess, and R. J. Newport, Phys. Rev. B 50, 846 (1994). 